\title{
Engaging Hospitalists in Antimicrobial Stewardship: Lessons From a Multihospital Collaborative
}

\author{
Megan R. Mack, MD*, Jeffrey M. Rohde, $\mathrm{MD}^{1}$, Diane Jacobsen, $M P H^{2}$, James R. Barron, $\mathrm{MD}^{3}$, Christin Ko, MD, MBA4, \\ Michael Goonewardene, MD, PhD5, David J. Rosenberg, MD, MPH, FACP, SFHM6, Arjun Srinivasan, MD, \\ Scott A. Flanders, MD, MHM ${ }^{1}$
}

\begin{abstract}
${ }^{1}$ Department of Internal Medicine, University of Michigan Hospital and Health System, Ann Arbor, Michigan; 2Institute for Healthcare Improvement, Cambridge, Massachusetts; ${ }^{3}$ Department of Internal Medicine, Spectrum Health System, Grand Rapids, Michigan; ${ }^{4}$ Department of Internal Medicine, Northwestern Memorial Hospital, Chicago, Illinois; ${ }^{5}$ Department of Internal Medicine, Reading Health System, West Reading, Pennsylvania; ${ }^{6}$ Department of Internal Medicine, Hofstra North Shore LIJ School of Medicine, Manhasset, New York; 'Division of Healthcare Quality Promotion, Centers for Disease Control and Prevention, Atlanta, Georgia.
\end{abstract}

Inappropriate antimicrobial use in hospitalized patients contributes to antimicrobial-resistant infections and complications. We sought to evaluate the impact, barriers, and facilitators of antimicrobial stewardship best practices in a diverse group of hospital medicine programs. This multihospital initiative included 1 community nonteaching hospital, 2 community teaching hospitals, and 2 academic medical centers participating in a collaborative with the Centers for Disease Control and Prevention and the Institute for Healthcare Improvement. We conducted multimodal physician education on best practices for antimicrobial use including: (1) enhanced antimicrobial documentation, (2) improved quality and accessibility of local clinical guidelines, and (3) a 72-hour antimicrobial "timeout." Implementation barriers included variability in physician practice styles, lack of awareness of stewardship importance, and overly broad interventions. Facilitators included engaging hospitalists, collecting real time data and providing performance feedback, and appropriately limiting the scope of interventions. In 2 hospitals, complete antimicrobial documentation in sampled medical records improved significantly (4\% to $51 \%$ and $8 \%$ to $65 \%, P<0.001$ for each comparison). A total of 726 antimicrobial timeouts occurred at 4 hospitals, and $30 \%$ resulted in optimization or discontinuation of antimicrobials. With careful attention to key barriers and facilitators, hospitalists can successfully implement effective antimicrobial stewardship practices. Journal of Hospital Medicine 2016;11:576-580. (c) 2016 Society of Hospital Medicine
Inappropriate antimicrobial use in hospitalized patients is a well-recognized driver for the development of drug-resistant organisms and antimicrobialrelated complications such as Clostridium difficile infection (CDI). ${ }^{1,2}$ Infection with $C$ difficile affects nearly 500,000 people annually resulting in higher healthcare expenditures, longer lengths of hospital stay, and nearly 15,000 deaths. ${ }^{3}$ Data from the Centers for Disease Control and Prevention (CDC) suggest that a $30 \%$ reduction in the use of broad-spectrum antimicrobials, or a $5 \%$ reduction in the proportion of hospitalized patients receiving antimicrobials, could equate to a $26 \%$ reduction in $\mathrm{CDI}^{4}{ }^{4}$ It is estimated that up to $50 \%$ of antimicrobial use in the hospital setting may be inappropriate. ${ }^{5}$

Since the Infectious Diseases Society of America and the Society for Healthcare Epidemiology of America published guidelines for developing formal, hospital-

\footnotetext{
*Address for correspondence and reprint requests: Megan R. Mack, MD Clinical Instructor, Hospitalist Program, 3119 Taubman Center, 1500 E. Medical Center Drive, SPC 5376, Ann Arbor, MI 48109; Telephone: 734647-0332; Fax: 734-232-9343; E-mail: megantre@med.umich.edu

Additional Supporting Information may be found in the online version of this article.

Received: November 25, 2015; Revised: March 11, 2016; Accepted: April 4, 2016

2016 Society of Hospital Medicine DOI 10.1002/jhm.2599

Published online in Wiley Online Library (Wileyonlinelibrary.com).
}

based antimicrobial stewardship programs in 2007, stewardship practices have been adapted by frontline providers to fit day-to-day inpatient care. ${ }^{5} \mathrm{~A}$ recent review by Hamilton et al. described several studies in which stewardship practices were imbedded into daily workflows by way of checklists, education reminders, and periodic review of antimicrobial usage, as well as a multicenter pilot of point-of-care stewardship interventions successfully implemented by various providers including nursing, pharmacists, and hospitalists. ${ }^{6}$

In response to the CDC's 2010 Get Smart for Healthcare campaign, which focused on stemming antimicrobial resistance and improving antimicrobial use, the Institute for Healthcare Improvement (IHI), in partnership with the CDC, brought together experts in the field to identify practical and feasible target practices for hospital-based stewardship and created a Driver Diagram to guide implementation efforts (Figure 1). Rohde et al. described the initial pilot testing of these practices, the decision to more actively engage frontline providers, and the $3 \mathrm{key}$ strategies identified as high-yield improvement targets: enhancing the visibility of antimicrobial use at the point of care, creating easily accessible antimicrobial guidelines for common infections, and the implementation of a 72 hour timeout after initiation of antimicrobials. ${ }^{7}$

In this article, we describe how, in partnership with the IHI and the CDC, the hospital medicine programs 


\section{Antibiotic Stewardship Driver Diagram}
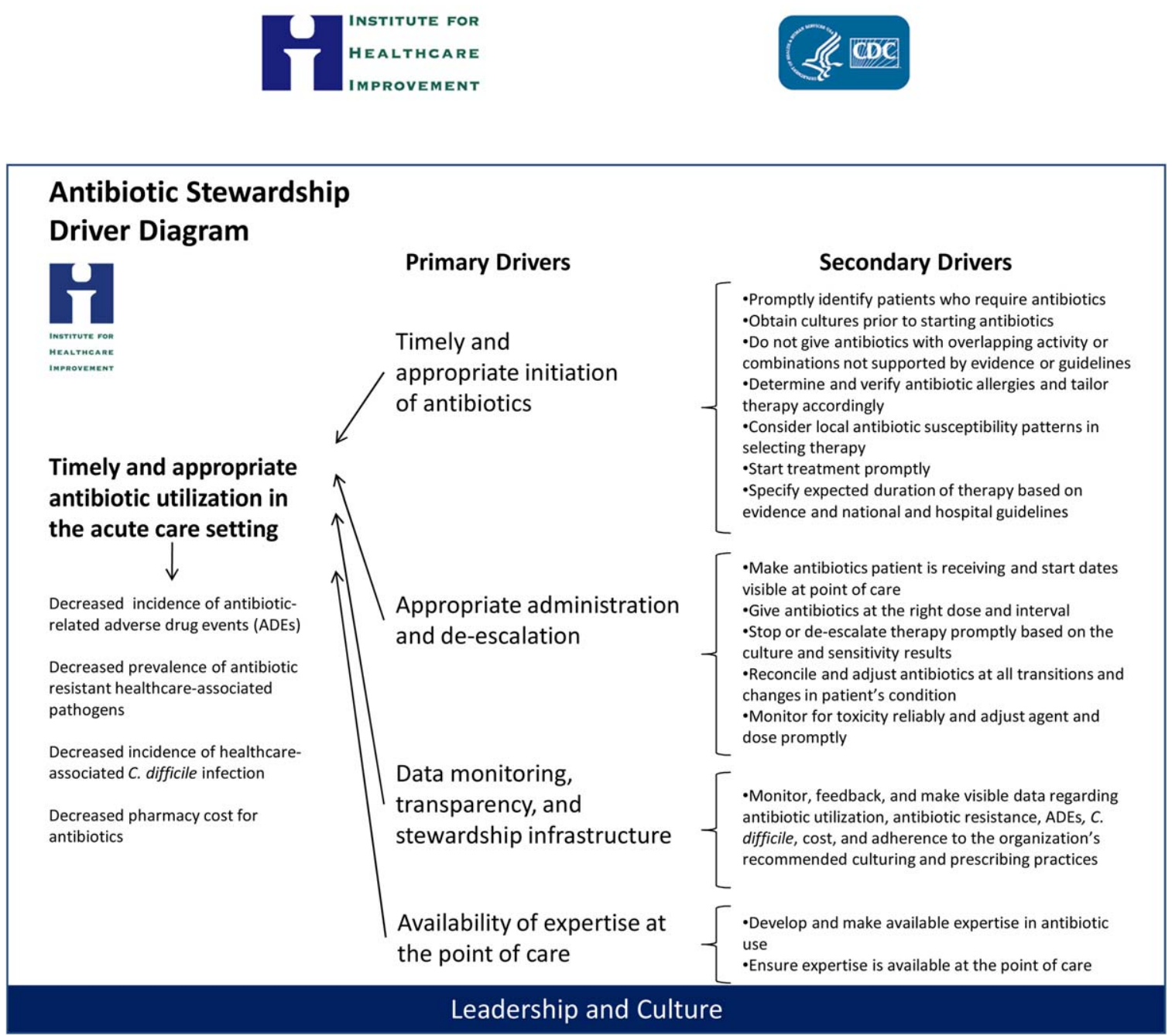

FIG. 1. Shown is the Antibiotic Stewardship Driver Diagram that was developed as part of the Centers for Disease Control and Prevention (CDC) and Institute for Healthcare Improvement partnered efforts to stem antimicrobial overuse through the CDC's Get Smart for Healthcare campaign. Eight pilot hospitals were recruited to participate in field testing and to refine the diagram in a variety of settings from September 2011 through June 2012.

at 5 diverse hospitals iteratively tested these 3 strategies with a goal of identifying the barriers and facilitators to effective hospitalist-led antimicrobial stewardship.

\section{METHODS}

Representatives from 5 hospital medicine programs, IHI, and the CDC attended a kick-off meeting at the CDC in November 2012 to discuss the 3 proposed strategies, examples of prior testing, and ideas for implementation. Each hospitalist provided a high-level summary of the current state of stewardship efforts at their respective institutions, identified possible future states related to the improvement strategies, and anticipated problems in achieving them. The $3 \mathrm{key}$ strategies are described below.

Improved Documentation/Visibility at Points of Care Making antimicrobial indication, day of therapy, and anticipated duration transparent in the medical record was the targeted improvement strategy to avoid unnecessary antimicrobial days that can result from provider uncertainty, particularly during patient handoffs. Daily hospitalist documentation was identified as a vehicle through which these aspects of antimicrobial use could be effectively communicated and propagated from provider to provider.

Stewardship educational sessions and/or awareness campaigns were hospitalist led, and were accompanied by follow-up reminders in the forms of emails, texts, flyers, or conferences. Infectious disease physicians were not directly involved in education but were available for consultation if needed.

\section{Improved Guideline Clarity and Accessibility}

Enhancing the availability of guidelines for frequently encountered infections and clarifying key guideline recommendations such as treatment duration were identified as the improvement strategies to help make 
treatment regimens more appropriate and consistent across providers.

Interventions included designing simplified pocket cards for commonly encountered infections, (see Supporting Information, Appendix A, in the online version of this article), collaborating with infectious disease physicians on guideline development, and dissemination through email, smartphone, and wall flyers, and creation of a continuous medical education module focused on stewardship practices.

\section{2-Hour Antimicrobial Timeout}

The 72-hour antimicrobial timeout required that hospitalists routinely reassess antimicrobial use 72 hours following antimicrobial initiation, a time when most pertinent culture data had returned. Hospitalists partnered with clinical pharmacists at all sites, and addressed the following questions during each timeout: (1) Does the patient have a condition that requires continued use of antimicrobials? (2) Can the current antimicrobial regimen be tailored based on culture data? (3) What is the anticipated treatment duration? A variety of modifications occurred during timeouts, including broadening or narrowing the antimicrobial regimen based on culture data, switching to an oral antimicrobial, adjusting dose or frequency based on patient-specific factors, as well as discontinuation of antimicrobials. Following the initial timeout, further adjustments were made as the clinical situation dictated; intermittent partnered timeouts continued during a patient's hospitalization on an individualized basis. Hospitalists were encouraged to independently review new diagnostic information daily and make changes as needed outside the dedicated time-out sessions. All decisions to adjust antimicrobial regimens were provider driven; no hospitals employed automated antimicrobial discontinuation without provider input.

\section{Implementation and Evaluation}

Each site was tasked with conducting small tests of change aimed at implementing at least 1, and ideally all 3 strategies. Small, reasonably achievable interventions were preferred to large hospital-wide initiatives so that key barriers and facilitators to the change could be quickly identified and addressed.

Methods of data collection varied across institutions and included anonymous physician survey, face-toface physician interviews, and medical record review. Evaluations of hospital-specific interventions utilized convenience samples to obtain real time, actionable data. Postintervention data were distributed through biweekly calls and compiled at the conclusion of the project. Barriers and facilitators of hospitalist-centered antimicrobial stewardship collected over the course of the project were reviewed and used to identify common themes.

\section{RESULTS}

Participating hospitals included 1 community nonteaching hospital, 2 community teaching hospitals, and 2 academic medical centers. All hospitals used computerized order entry and had prior quality improvement experience; 4 out of 5 hospitals used electronic medical records. Postintervention data on antimicrobial documentation and timeouts were compiled, shared, and successes identified. For example, 2 hospitals saw an increase in complete antimicrobial documentation from $4 \%$ and $8 \%$ to $51 \%$ and $65 \%$, respectively, of medical records reviewed over a 3month period. Additionally, cumulative timeout data across all hospitals showed that out of 726 antimicrobial timeouts evaluated, optimization or discontinuation occurred 218 times or $30 \%$ of the time.

Each site's key implementation barriers and facilitators were collected. Examples were compiled and common themes emerged (Table 1).

\section{DISCUSSION}

We successfully brought together hospitalists from diverse institutions to undertake small tests of change aimed at 3 key antimicrobial use improvement strategies. Following our interventions, significant improvement in antimicrobial documentation occurred at 2 institutions focusing on this improvement strategy, and 72-hour timeouts performed across all hospitals tailored antimicrobial use in $30 \%$ of the sessions. Through frequent collaborative discussions and information sharing, we were able to identify common barriers and facilitators to hospitalist-centered stewardship efforts.

Each participating hospital medicine program noticed a gradual shift in thinking among their colleagues, from initial skepticism about embedding stewardship within their daily workflow, to general acceptance that it was a worthwhile and meaningful endeavor. We posited that this transition in belief and behavior evolved for several reasons. First, each group was educated about their own, personal prescribing practices from the outset rather than presenting abstract data. This allowed for ownership of the problem and buy-in to improve it. Second, participants were able to experience the benefits at an individual level while the interventions were ongoing (eg, having other providers reciprocate structured documentation during patient handoffs, making antimicrobial plans clearer), reinforcing the achievability of stewardship practices within each group. Additionally, we focused on making small, manageable interventions that did not seem disruptive to hospitalists' daily workflow. For example, 1 group instituted antimicrobial timeouts during preexisting multidisciplinary rounds with clinical pharmacists. Last, project champions had both leadership and frontline roles within their groups and set the example for stewardship practices, which conveyed that this was a priority at the leadership level. These findings are in line with those of Charani et al., 
TABLE 1. Common Themes of Barriers and Facilitators to Antimicrobial Stewardship Within Each Hospitalist Program With Accompanying Examples

\begin{tabular}{|c|c|c|}
\hline \multirow{8}{*}{$\begin{array}{l}\text { Barriers: What impediments did we } \\
\text { experience during our stewardship project? }\end{array}$} & \multirow[t]{3}{*}{ Schedule and practice variability } & Physician variability in structure of antimicrobial documentation \\
\hline & & "Prescribing etiquette": it's difficult to change course of treatment plan started by a colleague \\
\hline & & Competing schedule demands of hospitalist and pharmacist \\
\hline & \multirow{3}{*}{$\begin{array}{l}\text { Skepticism of antimicrobial } \\
\text { stewardship importance }\end{array}$} & Perception of incorporating stewardship practices into daily work as time consuming \\
\hline & & "Improvement project fatigue" from competing quality improvement initiatives \\
\hline & & Unclear leadership buy-in \\
\hline & \multirow[t]{2}{*}{ Focusing too broadly } & Choosing large initial interventions, which take significant time/effort to complete and quantify \\
\hline & & Setting unrealistic expectations (eg, expecting "perfect" adherence to documentation, guidelines, or timeout) \\
\hline \multirow{10}{*}{$\begin{array}{l}\text { Facilitators: What countermeasures } \\
\text { did we target to overcome barriers? }\end{array}$} & \multirow[t]{4}{*}{ Engage the hospitalists } & Establish a core part of the hospitalist group as stewardship "champions" \\
\hline & & Speak 1-on-1 to colleagues about specific goals and ways to achieve them \\
\hline & & Establish buy-in from leadership \\
\hline & & Encourage participation from a multidisciplinary team (eg, bedside nursing, clinical pharmacists) \\
\hline & \multirow[t]{4}{*}{ Collect real time data and feedback } & Utilize a data collection tool if possible/engage hospital coders to identify appropriate diagnoses \\
\hline & & Define your question and identify baseline data prior to intervention \\
\hline & & Give rapid cycle feedback to colleagues that can impact antimicrobial prescribing in real time \\
\hline & & Recognize and reward high performers \\
\hline & \multirow[t]{2}{*}{ Limit scope } & Start with small, quickly implementable interventions \\
\hline & & Identify interventions that are easy to integrate into hospitalist workflow \\
\hline
\end{tabular}

NOTE: Barriers and facilitators were collected during biweekly conference calls as well as upon conclusion of our initiative.

who evaluated behavior change strategies that influence antimicrobial prescribing in acute care. The authors found that behavioral determinants and social norms strongly influence prescribing practices in acute care, and that antimicrobial stewardship improvement projects should account for these influences. ${ }^{8}$

We also identified several barriers to antimicrobial stewardship implementation (Table 1) and proposed measures to address these barriers in future improvement efforts. For example, hospital medicine programs without a preexisting clinical pharmacy partnership asked hospitalist leadership for more direct clinical pharmacy involvement, recognizing the importance of a physician-pharmacy alliance for stewardship efforts. To more effectively embed antimicrobial stewardship into daily routine, several hospitalists suggested standardized order sets for commonly encountered infections, as well as routine feedback on prescribing practices. Furthermore, although our simplified antimicrobial guideline pocket card enhanced access to this information, several colleagues suggested a smart phone application that would make access even easier and less cumbersome. Last, given the concern about the sustainability of antimicrobial stewardship initiatives, we recommended periodic reminders, random medical record review, and re-education if necessary on our 3 strategies and their purpose.

Our study is not without limitations. Each participating hospitalist group enacted hospital-specific interventions based on individual hospitalist program needs and goals, and although there was collective discussion, no group was tasked to undertake another group's initiative, thereby limiting generalizability. We did, however, identify common facilitators that could be adapted to a wide variety of hospitalist programs. We also note that our 3 main strategies were included in a recent review of quality indicators for measuring the success of antimicrobial stewardship programs; thus, although details of individual practice may vary, in principle these concepts can help identify areas for improvement within each unique stewardship program. ${ }^{9}$ Importantly, we were unable to evaluate the impact of the 3 key improvement strategies on important clinical outcomes such as overall antimicrobial use, complications including CDI, and cost. However, others have found that improvement strategies similar to our 3 key processes are associated with meaningful improvements in clinical outcomes as well as reductions in healthcare costs. ${ }^{10,11}$ Last, long- term impact and sustainability were not evaluated. By choosing interventions that were viewed by frontline providers as valuable and attainable, however, we feel that each group will likely continue current practices beyond the initial evaluation timeframe.

Although these 5 hospitalist groups were able to successfully implement several aspects of the 3 key improvement strategies, we recognize that this is only the first step. Further effort is needed to quantify the impact of these improvement efforts on objective patient outcomes such as readmissions, length of stay, and antimicrobial-related complications, which will better inform our local and national leaders on the 
inherent clinical and financial gains associated with hospitalist-led stewardship work. Finally, creative ways to better integrate stewardship activities into existing provider workflows (eg, decision support and automation) will further accelerate improvement efforts.

In summary, hospitalists at 5 diverse institutions successfully implemented key antimicrobial improvement strategies and identified important implementation facilitators and barriers. Future efforts at hospitalist-led stewardship should focus on strategies to scale-up interventions and evaluate their impact on clinical outcomes and cost.

\section{Acknowledgements}

The authors thank Latoya Kuhn, MPH, for her assistance with statistical analyses. We also thank the clinical pharmacists at each institution for their partnership in stewardship efforts: Patrick Arnold, PharmD, and Matthew Tupps, PharmD, MHA, from University of Michigan Hospital and Health System; and Roland Tam, PharmD, from Emory Johns Creek Hospital.

Disclosures: Dr. Flanders reports consulting fees or honoraria from the Institute for Healthcare Improvement, has provided consultancy to the Society of Hospital Medicine, has served as a reviewer for expert testimony, received honoraria as a visiting lecturer to various hospitals, and has received royalties from publisher John Wiley \& Sons. He has also received grant funding from Blue Cross Blue Shield of Michigan and the Agency for Healthcare Research and Quality. Dr. Ko reports consultancy for the American Hospital Association and the Society of Hospital Medicine involving work with catheter-associated urinary tract infections. Ms. Jacobsen reports grant funding from the Institute for Healthcare Improvement. Dr. Rosenberg reports consultancy for Bristol-Myers Squibb, Forest Pharmaceuticals, and Pfizer. The funding source for this collaborative was through the Institute for Healthcare Improvement and Centers for Disease Control and Prevention. Funding was provided by the Department of Health and Human Services, the Centers for Disease Control and Prevention, the National Center for Emerging Zoonotic and Infectious Diseases, and the Division of Healthcare Quality Promo-
tion/Office of the Director. Avaris Concepts served as the prime contractor and the Institute for Healthcare Improvement as the subcontractor for the initiative. The findings and conclusions in this report represent the views of the authors and might not reflect the views of the Centers for Disease Control and Prevention. The authors report no conflicts of interest.

\section{References}

1. Maragakis LL, Perencevich EN, Cosgrove SE. Clinical and economic burden of antimicrobial resistance. Expert Rev Anti Infect Ther. 2008;6(5):751-763.

2. Roberts RR, Hota B, Ahmad I, et al. Hospital and societal costs of antimicrobial-resistant infections in a Chicago teaching hospital: implications for antibiotic stewardship. Clin Infect Dis. 2009;49(8): $1175-1184$.

3. Lessa FC, Mu Y, Bamberg WM, et al. Burden of Clostridium difficile infection in the United States. N Engl J Med. 2015;372(9):825-834.

4. Fridkin S, Baggs J, Fagan R, et al.; Centers for Disease Control and Prevention (CDC). Vital signs: improving antibiotic use among hospitalized patients. MMWR Morb Mortal Wkly Rep. 2014;63(9):194200.

5. Dellit TH1, Owens RC, McGowan JE Jr, et al.; Infectious Diseases Society of America; Society for Healthcare Epidemiology of America. Infectious Diseases Society of America and the Society for Healthcare Epidemiology of America guidelines for developing an institutional program to enhance antimicrobial stewardship. Clin Infect Dis. 2007; 44(2):159-177.

6. Hamilton KW, Gerber JS, Moehring R, et al.; Centers for Disease Control and Prevention Epicenters Program. Point-of-prescription interventions to improve antimicrobial stewardship. Clin Infect Dis. 2015;60(8):1252-1258

7. Rohde JM, Jacobsen D, Rosenberg DJ. Role of the hospitalist in antimicrobial stewardship: a review of work completed and description of a multisite collaborative. Clin Ther. 2013;35(6):751-757.

8. Charani E, Edwards R, Sevdalis N, et al. Behavior change strategies to influence antimicrobial prescribing in acute care: a systematic review. Clin Infect Dis. 2011;53(7):651-662.

9. van den Bosch, Geerlings SE, Natsch S, Prins JM, Hulscher ME. Quality indicators to measure appropriate antibiotic use in hospitalized adults. Clin Infect Dis. 2015;60(2):281-291.

10. Bosso JA, Drew RH. Application of antimicrobial stewardship to optimise management of community acquired pneumonia. Int J Clin Pract. 2011;65(7):775-783.

11. Davey P, Brown E, Charani E, et al. Interventions to improve antibiotic prescribing practices for hospital inpatients. Cochrane Database Syst Rev. 2013;4:CD003543. 